
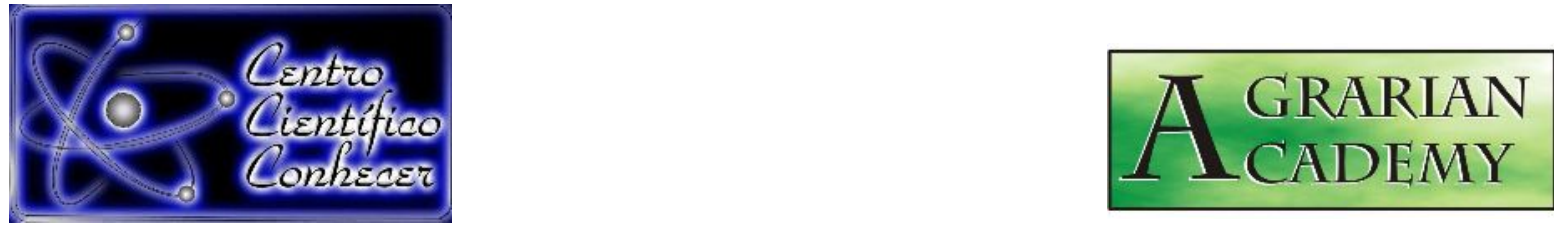

\title{
APLICAÇÕES DA TERMOGRAFIA NA AGRICULTURA
}

\author{
Francisco Fábio Lima Gomes ${ }^{1}$, Joilson Silva Lima ${ }^{2}$, Luis Gonzaga Pinheiro Neto ${ }^{3}$ \\ ${ }^{1}$ Aluno de Tecnologia em Irrigação e Drenagem do Instituto Federal de Educação, \\ Ciência e Tecnologia do Ceará, Sobral, Ceará, Brasil \\ ${ }^{2}$ Engenheiro Agrônomo do Instituto Federal de Educação, Ciência e Tecnologia do \\ Ceará, Sobral, Ceará, Brasil \\ ${ }^{3}$ Professor do Instituto Federal de Educação, Ciência e Tecnologia do Ceará, Sobral, \\ Ceará, Brasil (luis.neto@ifce.edu.br)
}

Recebido em: 15/12/2021 - Aprovado em: 15/12/2021 - Publicado em: 30/12/2021

DOI: 10.18677/Agrarian_Academy_2021B9

trabalho licenciado sob licença Creative Commons Attribution-NonCommercial-NoDerivatives 4.0 International License.

\begin{abstract}
RESUMO
A termografia é uma técnica que permite detectar a radiação infravermelha emitida pelos corpos, convertendo-a em imagens visíveis, as quais contêm informação sobre a temperatura e a superfície dos corpos. Dentre as técnicas do sensoriamento remoto, a termografia pode ser usada potencialmente na agricultura. $\mathrm{O}$ objetivo deste trabalho foi elaborar uma revisão de literatura mostrando aplicações da termografia na agricultura. A agricultura é uma área cada vez mais tecnológica, exemplo disso é o uso crescente de sistemas de detecção remota para monitorizar a condição fisiológica das culturas. Estes sistemas permitem detectar e registrar as assinaturas da radiação emitida pelas plantas, que podem ser relacionadas com diferentes parâmetros morfofisiológicos como biomassa, transpiração e fotossíntese. Por décadas, ficou bem estabelecido que o estresse hídrico das culturas pode ser detectado remotamente, medindo a temperatura da superfície das plantas, sendo considerada um importante indicador do nível real de estresse hídrico em uma cultura, constituindo-se uma ferramenta valiosa para o planejamento da irrigação. Plantas, após serem infectadas por patógenos sofrem danos que provocam mudanças na taxa de transpiração e no fluxo de água em toda a planta ou em órgãos da planta, levando ao aumento da temperatura em partes localizadas, tal como as folhas. Assim, termografia surge como uma ferramenta a ser utilizada na agricultura, com bons resultados no manejo da irrigação, sendo capaz de monitorar o estresse hídrico com base na temperatura da folha, mostrando-se também eficaz na detecção e no avanço de doenças em plantas e capaz de detectar, de forma remota e não invasiva, a condição fisiológica das culturas.
\end{abstract}

PALAVRAS-CHAVE: imagens térmicas, estresse hídrico, fisiologia vegetal. 


\title{
APPLICATIONS OF THERMOGRAPHY IN AGRICULTURE
}

\begin{abstract}
Thermography is a technique that allows the detection of infrared radiation emitted by bodies, converting it into visible images, which contain information about the temperature and surface of the bodies. Among remote sensing techniques, thermography can potentially be used in agriculture. The objective of this work was to elaborate a literature review showing applications of thermography in agriculture. Agriculture is an increasingly technological area, an example of which is the growing use of remote sensing systems to monitor the physiological condition of crops. These systems allow detecting and recording the signatures of radiation emitted by plants, which can be related to different morphophysiological parameters such as biomass, transpiration and photosynthesis. For decades, it has been well established that crop water stress can be remotely detected by measuring the surface temperature of plants, being considered an important indicator of the actual level of water in a crop, constituting a valuable tool for crop planning. irrigation. Plants, after being infected by pathogens suffer damage that causes changes in the rate of transpiration and water flow throughout the plant or in plant organs, leading to an increase in temperature in localized parts, such as the leaves. Thus, thermography emerges as a tool to be used in agriculture, with good results in irrigation management, being able to monitor water stress based on leaf temperature, being also effective in detecting and advancing diseases in plants and capable of remotely and non-invasively detecting the physiological condition of cultures.
\end{abstract}

KEYWORDS: thermal images, hydrical stress, plant physiology.

\section{INTRODUÇÃO}

O surgimento da termografia infravermelha está relacionada às observações feitas pelo médico, filósofo e pesquisador Grego Hipócrates, que comprovou variações da temperatura corporal por meio da alteração das propriedades da argila, utilizada como instrumento de avaliação. Mais tarde, Willians e John Herschel realizaram as primeiras imagens termais utilizando o sistema infravermelho por meio do método evaporográfico, o qual consiste na volatilização do álcool obtido de uma superfície pintada com carbono (MERINO et al., 2018).

Em 1950, os princípios da termologia no desenvolvimento de projetos de defesa militar foram aplicados pelo exército americano, buscando capturar informações de temperatura, utilizando câmeras de imagem infravermelha. Muitas das formas mais primitivas de medição de temperatura, tais como termômetros de contato e termometria de cristal líquido foram rapidamente substituídas por estes dispositivos modernos que, rapidamente, revolucionaram a ciência (CÔRTE; HERNANDEZ, 2016).

A termografia é uma técnica que permite detectar a radiação infravermelha emitida pelos corpos convertendo-a em imagens visíveis, as quais contêm informação sobre a temperatura e a superfície dos corpos (termogramas). Dentre as técnicas do sensoriamento remoto, a termografia pode ser usada potencialmente na agricultura e na fisiologia vegetal (BESSA, 2021).

A termografia é uma potencial ferramenta para se estimar o estresse hídrico, através do monitoramento da temperatura da copa da planta. Conforme relatam Meron et al. (2013), quando combinada com a análise automatizada de imagens, o uso de câmeras térmicas torna-se muito precisa, possibilitando a medição da temperatura relativa. 
A imagem térmica tem sido utilizada com grande potencial nas ciências agronômicas e ambientais, e também na agroindústria alimentícia. Imagens térmicas podem mostrar diferenças entre plantas com e sem estresse hídrico e até mesmo diferentes intensidades de irrigação. Em alguns casos, uma simples imagem pode ser suficiente para revelar variações espaciais no estado hídrico das plantas (SARAIVA et al., 2014).

Todos os processos bioquímicos e fisiológicos das plantas têm a participação da água, a qual é responsável, por exemplo, pela regulação térmica do vegetal (SARAIVA et al., 2014). Como a evaporação da água é um processo que demanda energia, na medida em que se aumentam as taxas de evapotranspiração, ocorre a diminuição da temperatura da superfície das folhas e plantas. Com isso, a temperatura da folha ou do dossel pode ser utilizada como indicador de estresse hídrico, principalmente para fins de manejo de áreas irrigadas e para avaliação do monitoramento do déficit hídrico (SILVEIRA et al., 2020).

As plantas, quando estressadas, tendem a fechar seus estômatos, diminuindo a taxa de transpiração e, consequentemente, levando ao aumento da temperatura foliar. Uma das formas de se obter informações sobre a temperatura do dossel é a utilização de câmeras digitais capazes de captarem imagens térmicas infravermelhas, imagens essas, que estão altamente correlacionadas com a condutância estomática e com o potencial hídrico do caule (FURLAM, 2017).

Outra aplicação da termografia está associada à detecção de doenças em plantas. Em estudos mais precisos, o sensoriamento remoto hiperespectral pode detectar mudanças sutis nas características biofísicas e bioquímicas das copas das plantas causadas por vários estresses, incluindo aqueles que são causados por patógenos, constituindo-se uma ferramenta valiosa para a detecção precoce de doenças de plantas, cuja não possam ser visualizadas a olho nu (ARANTES et al., 2019). Diante do exposto, o objetivo deste trabalho foi elaborar uma revisão de literatura sobre aplicações da termografia na agricultura.

\section{TERMOGRAFIA INFRAVERMELHA}

A radiação térmica emitida pode estar situada nas faixas de ultravioleta, visível, infravermelho e até na faixa de micro-ondas do espectro eletromagnético, em comprimentos que variam entre $760 \mathrm{~nm}$ e $1 \mathrm{~mm}$ (SANTOS, 2006). A descoberta da radiação infravermelha por Sir William Herschel em 1800 foi rapidamente seguida pela gravação da primeira imagem térmica por seu filho, John Herschel, que abriu novas dimensões no campo de medição de temperatura (LAHIRI et al., 2012).

A termografia infravermelha (IRT) surgiu como uma tecnologia na década de 1960 desenvolvida para os militares dos Estados Unidos, inicialmente para vigilância noturna e detecção de assinatura de calor, com a expansão do acesso a não militares, cientistas e civis, é agora amplamente usada em vários campos, incluindo aplicação na lei, engenharia, avaliação predial, na medicina, bem como na área biológica (TATTERSALL, 2016).

A termografia infravermelha é uma técnica de teste não destrutivo baseada no princípio de que dois materiais diferentes, ou seja, possuindo propriedades termo-físicas diferentes, produziriam duas assinaturas térmicas distintas que podem ser reveladas por um sensor infravermelho, como uma câmera térmica (CASTANEDO et al., 2013). Esse método é seguro, de baixo custo, e permite o registro rápido e não invasivo de radiação de energia que é liberada do corpo (CUEVAS et al., 2015), que possibilita a mensuração da temperatura de qualquer superfície ou material, contanto que sua temperatura encontre-se acima do zero 
absoluto (ALVES et al., 2020).

É uma ciência dedicada à aquisição e processamento termográfico com informações de dispositivos de medição sem contato. É baseada na radiação infravermelha (abaixo do vermelho), uma forma de radiação eletromagnética com comprimentos de onda mais longos do que aqueles da luz visível (USAMENTIAGA et al., 2014). Com o advento das novas gerações de câmeras infravermelhas, a IRT está se tornando uma técnica mais precisa, confiável e econômica (BAGAVATHIAPPAN et al., 2013).

Todo sistema com temperatura acima de $0 \mathrm{~K}$ emite radiação, cuja intensidade e distribuição espectral são determinadas pela temperatura do sistema (MAES; STEPPE, 2012). A radiação infravermelha emitida na faixa térmica de 8 a 12 $m$ pode ser detectada por câmeras termográficas e infravermelhas e é ilustrada em imagens em cores falsas, onde cada pixel da imagem contém o valor da temperatura do objeto medido (MAHLEIN, 2016).

Dispositivos de imagem ou câmeras térmicas usam predominantemente sensores de microbolômetro. Por serem dispositivos sensíveis, muitas vezes não podem ser instalados permanentemente no campo. Quando comparados com termômetros infravermelhos, são muito mais caros, porém, com a vantagem de fornecerem imagens e serem mais precisos (MAES; STEPPE, 2012).

A termografia por infravermelho é uma ferramenta de detecção aparentemente simples de ser manuseada (ALVES et al., 2020). Na ciência das plantas, a termografia pode ser usada em diferentes escalas temporais e espaciais, desde aplicações aéreas até aplicações em pequena escala (MAHLEIN, 2016). Entretanto, durante a avaliação, existem influências e limitações que podem sub ou superestimar o resultado (ALVES et al., 2020). Geralmente está sujeita a fatores ambientais, como temperatura ambiente, luz solar, chuva ou velocidade do vento (MAHLEIN, 2016). Como a ferramenta analisa a radiação térmica que atinge o seu detector, ambientes abertos ou até mesmo no interior de instalações, influências como radiação solar, vento, mudanças na temperatura ambiente, chuva, umidade excessiva, presença de partículas suspensas no ar, rochas, árvores e construções vizinhas podem estar constantemente emitindo radiação e comprometendo os resultados obtidos (ALVES et al., 2020).

\section{POTENCIAL HÍDRICO FOLIAR}

As plantas desenvolveram uma multiplicidade de formas morfológicas e fisiomecanismos de resposta lógica para resistir à seca. Dentre essas respostas, está a capacidade de manter processos fisiológicos no declínio do potencial de água da folha, e de evitar ou adiar essa diminuição do potencial durante a seca (FINI et al., 2013). Segundo Klein (2014), as árvores podem ajustar o uso da água e a segurança hídrica através da coordenação da regulação hidráulica e estomática.

Muitos aspectos morfológicos e anatômicos das folhas das plantas podem influenciar e controlar a perda de água para a atmosfera. Alguns exemplos são destacados como a presença de tricomas na superfície foliar, que atuam como quebra ventos microscópicos. Algumas plantas possuem os estômatos em cavidades, proporcionado abrigo externo à fenda estomática. Além disso, o tamanho e a forma da folha também influenciam na perda de água (TAIZ; ZEIGER, 2009).

Outra de suas estratégias é ter cutículas foliares mais expensas, que atuam como barreiras eficientes contra a perda de água após o fechamento estomático (YANG et al., 2016). Batista et al. (2010) analisaram o potencial de tolerância ao déficit hídrico em plantas de quinze diferentes cultivares de café arábica. Os 
resultados da anatomia foliar mostraram que a cultivar que exibiu alto potencial hídrico se encontrou entre as cultivares com maior espessura de cutícula de ambas as faces da epiderme, enquanto que a cultivar com as menores espessuras da cutícula se encontrou na faixa com menores potenciais hídricos.

A demanda de água com base nas plantas é comumente avaliada pela medição do potencial de água do caule ou da folha. Este último é muitas vezes preferido devido à sua natureza integrativa, permitindo um conjunto mínimo de locais de medição e devido à disponibilidade de ferramentas (câmaras de pressão) (ZOVKO et al.,2018). Durante períodos de pouca transpiração, o potencial de água na folha é frequentemente assumido como equilibrado com o potencial da água do solo. Durante os períodos diurnos diminui, para criar a força motriz necessária para mover a água do solo para as folhas (MOMEM et al., 2017).

Estudos de estresse hídrico na vegetação, muitas vezes consideram a dinâmica do potencial hídrico da folha $(\psi L)$, que há muito tempo é conhecido por fisiologistas de plantas por refletir o status hidráulico da planta e está fortemente ligado ao fechamento estomático e embolia do xilema (MOMEM et al., 2017). O potencial da água é negativo no xilema de praticamente todas as plantas terrestres. Ele é reduzido pela transpiração das folhas, auxiliada pelas forças coesivas entre as moléculas de água, fazendo com que a água fique sob tensão, ou seja, sob pressão negativa. As forças coesivas são equilibradas pela tensão superficial e o equilíbrio se manifesta como superfícies curvas e côncavas de ar-água nas folhas (VESALA et al., 2017). Devido às flutuações diurnas e sazonais no vapor folha-ar déficit de pressão, uma das principais funções regulatórias desempenhadas por estômatos é para limitar o déficit hídrico foliar induzido pela transpiração (DOMEC; JOHNSON, 2012).

As diferenças no controle estomático resultam em uma classificação de espécies de plantas em sua capacidade de manter o potencial hídrico da folha sob déficit hídrico. Um comportamento isohídrico é observado em espécies como milho ou choupo, com um fechamento estomático precoce que permite a manutenção do potencial da água da folha. Um comportamento anisohídrico é observado nas espécies como o girassol, que tem uma resposta estomática menos sensível, sofrendo assim um grande declínio no potencial da água da folha sob déficit hídrico (PARENT et al., 2010).

Um índice de estresse hídrico da cultura (CWSI) com base na temperatura da folha tem sido usado como indicativo de potencial hídrico foliar (LWP) em várias culturas, e as imagens térmicas podem ser uma ferramenta preciosa para mapear LWP em áreas de café irrigado por gotejamento intensivo, a fim de ajustar as necessidades de irrigação de acordo com variabilidade espacial da umidade do solo (COSTA et al., 2020).

Estudos com termografia por infravermelho para o diagnóstico do estresse hídrico em soja irrigada mostraram haver diferenças entre os tratamentos irrigados e aqueles em suspensão de irrigação antes do agravamento do estresse com o uso de imagens térmicas. Essas diferenças foram observadas antes mesmo das plantas exibirem uma maior perda da capacidade dissipativa de calor e maiores danos ao aparato fotossintético (SARAIVA et al., 2014).

As imagens térmicas são muito utilizadas e acopladas aos VANTs para monitorar o estresse hídrico em diversas culturas, uma vez que esse estresse promove o fechamento estomático, reduzindo a transpiração e o resfriamento evaporativo, causando o aumento da temperatura foliar (GAGO et al., 2015). Esses mesmos autores, estudando as relações entre o potencial hídrico da folha (LWP) de 
café com base no índice de estresse hídrico da cultura (CWSI), calculado por meio de um sensor infravermelho portátil e uma câmera térmica, analisando índices de correlação, precisão e exatidão. Observou-se que, os CWSI e LWP apresentaram boas correlações nos modelos lineares cúbico. Concluiu-se que o CWSI é um bom indicador do potencial hídrico de uma copa de café e pode ser uma alternativa para medições de LWP da câmara de Scholander.

Bellvert et al. (2014), mapearam o indicador do índice de estresse hídrico da cultura (CWSI), através de uma vinha utilizando imagiologia de ar a partir de um VANT, bem como comparando esses resultados com medições em terra de CWSI e potencial hídrico da folha $(\Psi \mathrm{L})$. Demonstraram a viabilidade do uso de imagens térmicas de alta resolução para gerar mapas CWSI que podem ser usados para o gerenciamento de irrigação de precisão, incorporando a variabilidade dentro de um vinhedo.

\section{TROCAS GASOSAS}

As plantas interagem com o ambiente circundante, por meio de processos de troca de carbono, água e energia, mantendo um equilíbrio que lhes permite crescer e adaptar-se a condições variáveis de crescimento. As trocas gasosas foliares (fluxos de $\mathrm{CO}_{2}$ e $\mathrm{H}_{2} \mathrm{O}$ ) em resposta ao meio ambiente desempenham um papel fundamental nesta adaptação, permitindo ganhos fotossintéticos e perda de água bem como a regulação da temperatura do dossel (COSTA et al., 2013b).

Um dos principais fatores que afetam as trocas gasosas é o estresse hídrico. Nesses casos, os estômatos fecham mais cedo do que deviam, e a troca gasosa entre a planta e a atmosfera reduz levando consideravelmente a rendimentos diminuídos (SURESH et al., 2010). Uma vez que, a troca gasosa através dos estômatos das folhas é um fenômeno fundamental, seu papel como principal porta entre a biosfera e a atmosfera acarreta grandes impactos nos ciclos globais de carbono e água (KLEIN, 2014).

Tombesi et al. (2015), em estudo sobre fechamento estomático em plantas

de Vitis vinifera enfrentando estresse hídrico, observaram que a condutância estomática medida ao meio-dia diminuiu durante o experimento. A maior diminuição de condutância estomática ocorreu entre os dias 5 e 8 quando caiu de cerca de $0,130 \mathrm{~mol} \mathrm{~m} 2 \mathrm{~s}-1$ para $0,008 \mathrm{~mol} \mathrm{~m} 2 \mathrm{~s}-1 \mathrm{em}$ ambas as cultivares. Da mesma forma, as taxas de assimilação líquida de $\mathrm{CO}_{2}$ seguiram um padrão semelhante no decorrer do trabalho.

As respostas das plantas à seca e a relação entre as trocas gasosas e o estado da água, que dependem da intensidade do déficit hídrico, assimilação metabólica de $\mathrm{CO}_{2}$ e forma biológica, são claramente complexas. Nos últimos anos, vários trabalhos enfocaram as trocas gasosas da planta e o status da água durante a seca, e os resultados mostraram a diminuição dessas, e do status da água em resposta à seca (YAN et al., 2016).

Chen et al. (2010), examinando as respostas do desempenho fotossintético ao estresse hídrico e recuperação, em mudas de seringueira, observaram que os valores de taxa de assimilação fotossintética líquida máxima ( $A$ max) em plantas com estresse hídrico caiu abruptamente de 12,0 para 3,0 $\mu \mathrm{mol} \mathrm{CO} 2 \mathrm{~m}-2 \mathrm{~s}-1$ após 11-35 dias de retenção de água. Após 5 dias de reidratação, o valor de $A$ max em plantas anteriormente com estresse hídrico ainda era significativamente menor do que o valor de controle.

A agricultura é uma área cada vez mais tecnológica. Exemplo disso é o uso crescente de sistemas de detecção remota para monitorizar a condição fisiológica 
das culturas. Estes sistemas permitem detectar e registar as assinaturas da radiação emitida pelas culturas, por exemplo na zona do visível e dos infravermelhos, que podem ser relacionadas com diferentes parâmetros morfofisiológicos como: biomassa, transpiração e fotossíntese (COSTA et al., 2013a).

As medições das trocas gasosas nas folhas proporcionam informações diretas e normalmente precisas sobre a condição fisiológica e a variabilidade genética das plantas, especialmente em termos da atividade fotossintética e transpiracional das culturas em situações de estresse hídrico (TEJERO et al., 2015). Essas medições, envolvem contato com folhas e frequentemente interferem no funcionamento da mesma. Além disso, eles são demorados e podem ser limitados pela dimensão reduzida das amostras de folhas e/ou o grande número de medições a ser feito. Portanto, um método mais rápido, remoto e não invasivo de análises de rendimento é com base em imagens térmicas (COSTA et al., 2013b).

\section{USO DA TERMOGRAFIA NO MANEJO DA IRRIGAÇÃO}

A água é um dos recursos mais importantes que existem no planeta. Portanto, é necessário melhorar sua eficiência no uso, quer pela aplicação de métodos de irrigação mais eficientes, ou determinando as necessidades hídricas da cultura usando métodos precisos, como o da temperatura da folha para 0 monitoramento do estresse hídrico (ZIA et al., 2012). O uso da temperatura da folha ou do dossel como um indicador do estado da água da planta medido por câmeras infravermelhas ou termômetros é baseado no princípio de que o fechamento estomático das plantas ocorre durante o estresse hídrico, o que resulta em uma diminuição da dissipação de energia e aumento da temperatura do dossel (ZIA et al., 2012).

Por décadas, ficou bem estabelecido que o estresse hídrico das culturas pode ser detectado remotamente medindo a temperatura da superfície das plantas cultivadas, sendo considerada um importante indicador do nível real de estresse hídrico em uma planta, constituindo-se uma ferramenta valiosa para o planejamento da irrigação (PADHI et al., 2012). A vantagem potencial das imagens térmicas (também conhecido como termografia infravermelha) é a capacidade da imagem para cobrir um grande número de folhas e plantas de uma só vez em uma alta resolução espacial (ZIA et al., 2011; PADHI et al., 2012;).

Saraiva et al. (2014), trabalhando com imagens térmicas para avaliar o estresse hídrico severo em plantas de soja, obteve resultados satisfatórios. $O$ experimento mostrou que a partir de um dia sem irrigação, as plantas apresentaram temperaturas médias do solo dos vasos significativamente maiores em relação ao controle e que, as temperaturas foliares médias mantiveram-se maiores nas mudas sem irrigação $\left(3^{\circ} \mathrm{C}\right.$ acima das médias das plantas controle), comprovando a eficácia do uso de imagens térmicas para monitorar o status de água das plantas.

Aragão (2019), estudando a utilização de imagens termométricas na estimativa do estado hídrico do meloeiro, observou que o uso de imagens infravermelhas foi eficiente na identificação do estado hídrico dessa cultura, podendo ser empregada como estratégia de manejo da irrigação. Segundo o autor, a produtividade do meloeiro aumentou com a redução do índice térmico e as maiores lâminas de irrigação possibilitaram menores temperaturas do dossel.

O uso da termografia infravermelha (IRT) para determinar o estresse hídrico é um método sem contato e, portanto, rápido e prático (ZIA et al., 2011). Além de ser uma metodologia não invasiva usada para monitorar as características físicas e fisiológicas de plantas e para avaliar os efeitos dos estresses ambientais sobre o 
desempenho da cultura. De uma forma funcional, a imagem permite observações em diferentes escalas (desde uma única folha a plantas inteiras) (COSTA et al., 2013b). Segundo Saraiva et al. (2014), o uso de imagens térmicas é uma ferramenta não destrutiva eficiente de monitoramento e manejo hídrico das plantas.

A imagem térmica surgiu como uma técnica promitente não invasiva para controlar remotamente e de forma não destrutiva o estado da água da cultura com base na relação inversa entre a temperatura da folha e a taxa de transpiração. Esta técnica permite a exposição gráfica da distribuição espacial da temperatura da superfície da planta por meio da percepção da radiação infravermelha emitida e refletida pela cultura (TEJERO et al., 2015). Esses mesmos autores, testaram a resistência de diferentes genótipos de milho ao estresse hídrico com base em medições de imagem térmica e concluiu que esse método é uma abordagem adequada e rápida para monitorar a condição fisiológica de mudas de milho e pode funcionar como um parâmetro indicador de sensibilidade ao estresse hídrico e do estado da água das plantas.

A termografia quantitativa abre a possibilidade de monitoramento espaçotemporal rápido e não destrutivo da detecção de estresse hídrico em plantas usando a temperatura das folhas. Dados de imagem térmica, obtidos de fotografias aéreas de uma cultura com uma câmera térmica digital, provaram ser valioso na programação da irrigação, aumentando a eficiência do uso da água (ZOVKO et al., 2018).

Nos últimos anos, devido às melhorias nas tecnologias, e redução no custo dos sensores, a termologia ganhou popularidade. Ao fornecer medições de temperatura de solo e de superfícies de cultivo, os sensores térmicos têm potencial para serem usados em várias aplicações, incluindo monitoramento de estresse hídrico nas culturas e no solo, bem como, para planejamento e programação da irrigação (KHANAL et al., 2017).

Liu et al. (2020), examinando o potencial do uso de índices térmicos baseados em medições de temperatura do dossel para detectar estresse hídrico em plantas em ambiente natural, concluiram que, o índice térmico é recomendado com desempenho significativamente melhor para a avaliação do estresse hídrico para todas as árvores da amostra. Segundo esses mesmos autores, o meio-dia é o melhor horário para usar os índices térmicos como indicador de estresse hídrico da planta, devido à maior parte dos estômatos estarem fechados, causando o aumento da temperatura foliar.

\section{TERMOGRAFIA PARA DETECÇÃO DE DOENÇAS DE PLANTAS}

Imagens termográficas são uma forma de detecção precisa de pontos problemáticos, devido a temperatura da planta estar correlacionada com o balanço hídrico e a transpiração, que são afetadas por doenças (ARANTES et al., 2019). Trabalhos científicos relatam, que plantas após serem infectadas por patógenos sofrem danos que provocam mudanças na taxa de transpiração e fluxo de água em toda a planta ou em órgãos da planta. Isto leva ao aumento da temperatura em partes localizadas, tal como as folhas. Diante disto, imagens geradas por câmeras térmicas constituem uma forma de detectar presenças de infecção por patógeno ainda não visível a olho nu (VIANA et al., 2018; VIANA et al., 2019).

Ruiz et al. (2013), estudando uma nova abordagem de imagens aéreas de alta resolução para detecção da Huanglongbing (HLB) ou doença do greening dos citros, usando um veículo aéreo não tripulado (VANT) de sensor remoto de baixa altitude e multirotor de baixo custo, observaram que dentre os algoritmos de 
classificação testados, a máquina de vetores de suporte (SVM) com kernel resultou em melhor desempenho do que outros métodos como SVM (linear), análise discriminante linear e análise discriminante quadrática e concluíram que, o sensoriamento aéreo de alta resolução tem boas perspectivas para a detecção de árvores infectadas com HLB.

Calderón et al. (2014), explorando o uso de imagens térmicas e multiespectrais de alta resolução como um indicador de infecção por míldio obtiveram resultados que demonstram a viabilidade em detectar a infecção por Peronospora arborescens em plantas de papoula do ópio usando imagens térmicas e multiespectrais de alta resolução adquiridas com um veículo aéreo não tripulado (VANT).

Arantes et al. (2019), em pesquisa embasada na utilização do sensoriamento remoto através de imagem multiespectral de alta resolução espacial (RapidEye) para detectar, discriminar e espacializar a possível ocorrência de ferrugem na soja em distintos níveis de gravidade, concluíram que, a partir das cartas geradas foi possível caracterizar espacialmente as áreas de ferrugem em estágio intermediário e avançado da infecção, mesmo considerando a inexistência de pontos de verificação em campo. Entretanto a metodologia aplicada, não foi capaz de detectar o patógeno de uma forma precoce.

Para um estudo eficaz de imagens termográficas, a heterogeneidade entre e dentro das folhas pode ser utilizada. A diferença média de temperatura dentro de folhas isoladas, plantas e talhões de cultivo é um indicador importante para o aparecimento de doenças em plantas (MAHLEIN, 2016). Oerke et al. (2011), em trabalho sobre avaliação termográfica da sarna em folhas de macieira observaram que, as medidas termográficas revelaram diferenças na gravidade da doença resultante do estágio da moléstia, resistência do tecido do hospedeiro e diferenças na agressividade dos isolados de Venturia inaequalis.

\section{CONSIDERAÇÕES FINAIS}

A termografia é uma ferramenta que pode ser utilizada na agricultura com bons resultados no manejo da irrigação, sendo capaz de monitorar o estresse hídrico com base na temperatura da folha. $O$ uso de imagens termográficas mostra-se eficaz na detecção e no avanço de doenças em plantas. Além de ser capaz de detectar, de forma remota e não invasiva, a condição fisiológica das culturas.

\section{REFERÊNCIAS}

ALVES, F. V.; KARVATTE JUNIOR, N.; OLIVEIRA, C. C. Aplicações da termografia por infravermelho (TIV) na bovinocultura de corte. Campo Grande: Embrapa Gado de Corte, 2020. 36 p. (Documentos, 276). Disponível em: $<$ https://ainfo.cnptia.embrapa.br/digital/bitstream/item/214175/1/DOC-276-Final-emAlta.pdf>.

ARAGÃO, M. F. Utilização de imagens termométricas na estimativa do estado hídrico do meloeiro cultivado em solo com e sem cobertura vegetal. $2019.74 \mathrm{f}$. Dissertação (Mestrado em Engenharia Agrícola) - Centro de Ciências Agrárias, Universidade Federal do Ceará, Fortaleza. Disponível em: $<$ https://repositorio.ufc.br/bitstream/riufc/47001/3/2019_dis_mfaragao.pdf>.

ARANTES, B. H. T.; MARTINS, G. D.; CARVALHO, E. R.; NOGUEIRA, L. C. A. Identificação de ferrugem na soja por meio de imagens de alta resolução espacial. 
Revista Brasileira de Geografia Física, v. 12, n. 3, p. 1003-1016, 2019. Disponível em: <https://periodicos.ufpe.br/revistas/rbgfe/article/view/238815>. doi: 10.26848/rbgf.v12.3.p1003-1016

BAGAVATHIAPPAN, S.; LAHIRI, B. B.; SARAVANAN, T.; PHILIP, J.; JAYAKUMAR, T. Infrared thermography for condition monitoring - A review. Infrared Physics \& Technology, v. $60, \quad$ p. $35-55, \quad 2013$. Disponível em: <https://www.sciencedirect.com/science/article/pii/S1350449513000327>. doi: 10.1016/j.infrared.2013.03.006

BATISTA, L. A.; GUIMARÃES, R. J.; PEREIRA, F. J.; CARVALHO, G. R.; CASTRO, E. M. Anatomia foliar e potencial hídrico na tolerância de cultivares de café ao estresse hídrico. Revista Ciência Agronômica, v. 41, n. 3, p. 475-481, 2010. Disponível em: <https://www.scielo.br/j/rca/a/Tk8sjTB6TzW6yzZtLKtsB8z/?lang=pt>. doi: 10.1590/S1806-66902010000300022

BELLVERT, J.; TEJADA, P. J.; GIRONA, J.; FERERES, E. Mapping crop water stress index in a 'Pinot-noir' vineyard: comparing ground measurements with thermal remote sensing imagery from an unmanned aerial vehicle. Precision Agriculture, v. 15, p. 361-376, 2014. Disponível em: <https://link.springer.com/article/10.1007/s11119-013-9334-5>. doi: 10.1007/s11119013-9334-5

BESSA, R. T. Termografia na avaliação da temperatura de mudas de Tagetes erecta cultivadas em diferentes ambientes com e sem hidroretentor. 2021. $54 \mathrm{f}$. Monografia (Graduação em Agronomia) - Centro de Ciências Agrárias, Universidade Federal do Ceará, Fortaleza. Disponível em: <http://repositorio.ufc.br/bitstream/riufc/58879/3/2021_tcc_rtbessa.pdf>.

CALDERÓN, R.; BORREGO, M. M.; LANDA, B .B.;CORTÉS, J. A. N.; TEJADA, P. J. Z. Detection of downy mildew of opium poppy using high-resolution multi-spectral and thermal imagery acquired with an unmanned aerial vehicle. Precision Agriculture, v. 15, n. 6, p. 639-661, 2014. Disponível em: <https://link.springer.com/article/10.1007\%2Fs11119-014-9360-y>. doi: 10.1007/s11119-014-9360-y

CASTANEDO, C. I.; TARPANI, J. R.; MALDAGUE, X. P. Nondestructive testing with thermography. European Journal of Physics, v. 34, p. S91, 2013. Disponível em: <https://iopscience.iop.org/article/10.1088/0143-0807/34/6/S91>. doi: 10.1088/01430807/34/6/S91

CÔRTE, A. C. R.; HERNANDEZ, A. J. Termografia médica infravermelha aplicada à medicina do esporte. Revista Brasileira de Medicina do Esporte, v. 22, n. 4, p. 315-319, 2016. Disponível em: <https://www.scielo.br/j/rbme/a/CxYgWLRqL4YKZsPR757NyVC/?format=pdf\&lang=p t>. doi: 10.1590/1517-869220162204160783

COSTA, J. D. O; COELHO, R. D.; BARROS, T. H. S.; JUNIOR, E. F.; FERNANDES, A. L. T. Canopy thermal response to water deficit of coffee plants under drip irrigation. 
Irrigation and Drainage, v. 69, n. 3, p. 472-482, 2020. Disponível em: <https://onlinelibrary.wiley.com/doi/10.1002/ird.2429>. doi: 10.1002/ird.2429

COSTA, J. M.; IVÁN, F.; TEJERO, F. G.; CHAVES, M. O uso da termografia na agricultura moderna. Revista da Associação Portuguesa de Horticultura, n. 113, p. 30-34, 2013a. Disponível em: <https://www.researchgate.net/publication/282943605_O_uso_da_termografia_na_a gricultura_moderna>.

COSTA, J. M.; GRANT, O. M.; CHAVES, M. M. Thermography to explore plantenvironment interactions. Journal of Experimental Botany, n. 64, v. 13, p. 39373949, 2013b. Disponível em: <https://academic.oup.com/jxb/article/64/13/3937/435734>. doi: 10.1093/jxb/ert029

CHEN, J. W.; ZHANG, Q.; LI, X. S.; CAO, K. F. Gas exchange and hydraulics in seedlings of Hevea brasiliensis during water stress and recovery. Tree Physiology, v. 30, n. 7, p. 876-885, 2010. Disponível em: <https://pubmed.ncbi.nlm.nih.gov/20516484/>. doi: 10.1093/treephys/tpq043

CUEVAS, F. I.; MARINS, J. C. B.; LASTRAS, J. A.; CARMONA, P. M. G.; CANO, S. P.; CONCEPCIÓN, M. A.; QUINTANA, M. S. Classification of factors influencing the use of infrared thermography in humans: A review. Infrared Physics \& Technology, v. 71, p. 28-55, 2015. Disponível em: <https://www.sciencedirect.com/science/article/abs/pii/S1350449515000523>. doi: 10.1016/j.infrared.2015.02.007

DOMEC, J. C.; JOHNSON, D. M. Does homeostasis or disturbance of homeostasis in minimum leaf water potential explain the isohydric versus anisohydric behavior of Vitis vinifera L. cultivars?Tree Physiology, v. 32, n. 3, p. 245-248, 2012. Disponível em: <https://academic.oup.com/treephys/article/32/3/245/1688138>. doi: 10.1093/treephys/tps013

FINI, A.; BELLASIO, C.; POLLASTRI, S.; TATTINI, M.; FERRINI, F. Water relations, growth, and leaf gas exchange as affected by water stress in Jatropha curcas. Journal of Arid Environment, v. 89, p. 21-29. 2013. Disponível em: <https://www.sciencedirect.com/science/article/abs/pii/S0140196312002674>. doi: 10.1016/j.jaridenv.2012.10.009

FURLAN, D. A. Coeficiente de estresse hídrico utilizando termografia infravermelha-estudo em cafeeiro conilon (Coffea canephora). 2017. $56 \mathrm{f}$. Dissertação (Mestrado em Produção Vegetal) - Centro de Ciências e Tecnologias Agropecuárias, Universidade Estadual do Norte Fluminense Darcy Ribeiro, Campos dos Goytacazes. Disponível em: <https://uenf.br/posgraduacao/producao-vegetal/wpcontent/uploads/sites/10/2017/07/Disserta\%C3\%A7\%C3\%A3o-Diego-Furlan-FinalCD.pdf>.

GAGO, J.; DOUTHE, C.; COOPMAN, R. E.; GALLEGO, P. P.; CARBO, M. R.; et al.; challenge to assess water stress for sustainable agriculture. Agricultural Water Management, v. 153, p. 9-19, 2015. Disponível em: <https://www.sciencedirect.com/science/article/abs/pii/S0378377415000293\#: :text= 
UAVs\%20can\%20improve\%20temporal\%20and\%20spatial\%20sensing\%20resolutio ns\%20for\%20water\%20estresse\%20management.\&text=Multi\%2Dspectral\%20and\% 20thermal\%20images,water\%20estresse\%20in\%20several\%20crop.\&text=Easy\%20 and\%20low\%2Dcost\%20retrieving,UAVs\%20is\%20an\%20important\%20challenge. . . doi: 10.1016/j.agwat.2015.01.020

KHANAL, S.; FULTON, J.; SHEARER, S. An overview of current and potential applications of thermal remote sensing in precision agriculture.Computers and Electronics in Agriculture, v. 139, p. 22-32, 2017. Disponível em: $<$ https://www.cabdirect.org/cabdirect/abstract/20173228561>. 10.1016/j.compag.2017.05.001

KLEIN, T. The variability of stomatal sensitivity to leaf water potential across tree species indicates a continuum between isohydric and anisohydric behaviours. Functional Ecology, v. 28, n. 6, p. 1313-1320, 2014. Disponível em: <https://besjournals.onlinelibrary.wiley.com/doi/full/10.1111/1365-2435.12289>. doi: $10.1111 / 1365-2435.12289$

LAHIRI, B. B.; BAGAVATHIAPPAN, S.; JAYAKUMAR, T.; PHILIP, J. Medical applications of infrared thermography: a review. Infrared Physics \& Technology, v. 55, p. 221-235, 2012. Disponível em: <https://www.sciencedirect.com/science/article/pii/S1350449512000308>. doi: 10.1016/j.infrared.2012.03.007

LIU, N.; DENG, Z.; WANG, H.; LUO, Z.; HUGO A.; JURADO,G.;HE, X.; GUAN, H. Thermal remote sensing of plant water stress in natural ecosystems. Forest Ecology and Management, v. 476, 118433, 2020. Disponível em: <https://www.sciencedirect.com/science/article/abs/pii/S0378112720312020?via\%3Di hub>. doi: 10.1016/j.foreco.2020.118433

MAES, W. H.; STEPPE, K. Estimating evapotranspiration and drought stress with ground-based thermal remote sensing in agriculture: a review. Journal of Experimental Botany, n. 63, v. 13, 4671-4712, 2012. Disponível em: <https://academic.oup.com/jxb/article/63/13/4671/486348>. doi.org/10.1093/jxb/ers165

MAHLEIN, A. K. Plant disease detection by imaging sensors - parallels and specific demands for precision agriculture and plant phenotyping. Plant Disease, v. 100, n. 2, p. 241-251, 2016. Disponível em: <https://apsjournals.apsnet.org/doi/10.1094/PDIS03-15-0340-FE>. doi: 10.1094/PDIS-03-15-0340-FE

MERINO, E. A. D.; FORCELINI, F.; VARNIER, T.; MERINO, G. S. A. D. O uso da instrumentação tecnológica em projetos de tecnologia assistiva: captura de movimentos e termografia infravermelha. Human Factors in Design, v. 7, n. 14, p. 95-113, 2018. Disponível em: <https://www.revistas.udesc.br/index.php/hfd/article/view/2316796307142018095>. doi: $10.5965 / 2316796307142018095$

MERON, M.; SPRINTSIN, M.; TSIPRIS, J.; ALCHANATIS, V.; COHEN, Y. Foliage 
temperature extraction from thermal imagery for crop water stress determination. Precision Agriculture, v. 14, p. 467-477, 2013. Disponível em: <https://link.springer.com/article/10.1007\%2Fs11119-013-9310-0>. doi: 10.1007/s11119-013-9310-0

MOMEN, M.; WOOD, J. D.; NOVICK, K. A.; PANGLE, R.; POCKMAN, W. T.; MCDOOWELL, N. G.; KONINGS, A. G. Interacting effects of leaf water potential and biomass on vegetation optical depth. Journal Geophysical Resourch Biogeosciences, v. 122, n. 11, p. 3031-3046, 2017. Disponível em: <https://agupubs.onlinelibrary.wiley.com/doi/10.1002/2017JG004145>. /10.1002/2017JG004145

OERKE, E. C.; FRÖHLING, P.; ESTEINER, U. Thermographic assessment of scab disease on apple leaves. Precision Agriculture, v. 12, p. 699-715, 2011. Disponível em: <https://link.springer.com/article/10.1007\%2Fs11119-010-9212-3>. doi: $10.1007 / \mathrm{s} 11119-010-9212-3$

PADHI, J.; MISRA, R. K.; PAYERO, J. O. Estimation of soil water deficit in anirrigated cotton field within frared thermography. Field Crops Research, v. 126, p. 45-55, 2012. <https://www.sciencedirect.com/science/article/abs/pii/S0378429011003303>. doi: 10.1016/j.fcr.2011.09.015

PARENT, B.; SUARD, B.; SERRA, J. R.; TARDIEU, F. Rice leaf growth and water potential are resilient to evaporative demand and soil water deficit once the effects of root system are neutralized. Plant, Cell and Environment, 33, n. 8, p. 1256-1267, 2010. Disponível em: <https://pubmed.ncbi.nlm.nih.gov/20302604/>. doi: 10.1111/j.1365-3040.2010.02145.x

RUIZ, F. G.; SANKARAN, S.; MAJA J. M, LEE, W.S.; RASMUSSEN, J.; EHSANI, R. Comparison of two aerial imaging platforms for identification of Huanglongbinginfected citrus trees. Computers and Electronics in Agriculture, v. 91, p. 106-115, $2013 . \quad$ Disponível em: <https://www.sciencedirect.com/science/article/abs/pii/S0168169912002748>. doi: 10.1016/j.compag.2012.12.002

SANTOS, L. Termografia infravermelha em subestações de alta tensão desabrigadas. 2006. 129 f. Dissertação (Mestrado em Engenharia da Energia) Universidade Federal de Itajubá, Itajubá. Disponível em: $<$ https://www.researchgate.net/publication/276293466_TERMOGRAFIA_INFRAVER MELHA_em_Subestacoes_de_Alta_Tensao_Desabrigadas>.

SARAIVA, G.; ANDRADE, R.; SOUZA, G. Termografia por infravermelho como ferramenta de diagnóstico precoce de estresse hídrico severo em soja. Agrarian Academy, v. 1, n. 2, p. 158-169, 2014. Disponível em: <http://www.conhecer.org.br/Agrarian\%20Academy/2014b/termografia.pdf>.

SILVEIRA, J. M. C.; CÂNDIDO, B. M.; SILVA, A. L. B. O.; PAVÃO, G. C.; XAVIER, M. A.; PIRES, R. C. M. Uso de imagens multiespectrais e termográficas para monitoramento das condições hídricas da cana-de-açucar. Irriga, v. 25, n. 4, p. 689- 
696

2020.

Disponível

em:

<https://revistas.fca.unesp.br/index.php/irriga/article/view/4174>.

doi:

10.15809/irriga.2020v25n4p689-696

SURESH, K.; NAGAMANI, C.; RAMACHANDRUDU, K.; MATHUR, R. K. Gasexchange characteristics, leaf water potential and chlorophyll a fluorescence in oil palm (Elaeis guineensis Jacq.) seedlings under water stress and recovery. Photosynthetica, v. 48, n. 3, p. 430-436, 2010. Disponível em: $<$ https://ps.ueb.cas.cz/artkey/phs-201003-0016_gas-exchange-characteristics-leafwater-potential-and-chlorophyll-a-fluorescence-in-oil-palm-elaeis-guineensi.php>. doi: $10.1007 / \mathrm{s} 11099-010-0056-x$

TAIZ, L.; ZEIGER, E. Fisiologia Vegetal. 4. Ed. Porto Alegre: Artmed, 2009. 848 p.

TATTERSALL, G. J. Infrared thermography: A non-invasive window into thermal physiology. Comp Biochem Physiol A Mol Integr Physiol, v. 202, p. 78-98, 2016. Disponível em: <https://pubmed.ncbi.nlm.nih.gov/26945597/>. doi: 10.1016/j.cbpa.2016.02.022

TEJERO, I. V. G.; COSTA, J. M; LIMA, R. S. N.; ZUAGO, V. H. D.; CHAVES, M. M.; PATTO, M. C. V. Thermal imaging to phenotype traditional maize landraces for drought tolerance. Comunicata Scientiae, v. 6, n. 3, p. 334-343, 2015. Disponível em: <https://www.comunicatascientiae.com.br/comunicata/article/view/960>. doi: $10.14295 /$ cs.v6i3.960

TOMBESI, S.; NARDINI, A.; FRIONI, T.; SOCCOLINI, M.; ZADRA, C.; FARINELLI, D.; PONI, S.; PALLIOTTI,A. Stomatal closure is induced by hydraulic signals and maintained by ABA in drought-stressed grapevine. Scientific Reports, v. 5, 12449, 2015. Disponível em: <https://www.ncbi.nlm.nih.gov/pmc/articles/PMC4513549/>. doi: $10.1038 /$ srep12449

USAMENTIAGA, R.; VENEGAS, P.; GUEREDIAGA, J.; VEGA, L.; MOLLEDA, J.; BULNES, F. G. Tnfrared thermography for temperature measurement and nondestructive testing. Sensors, v. 14, p. 12305-12348, 2014. Disponível: <https://www.ncbi.nlm.nih.gov/pmc/articles/PMC4168422/>.

doi: $10.3390 /$ s 140712305

VESALA, T.; SEVANTO, S.; GRÖNHOLM, T.; SALMON, Y.; NIKINMAA, E.; HARI, P.; HÖLTTÄ, T. Effect of leaf water potential on internal humidity and CO2 dissolution: reverse transpiration and improved water use efficiency under negative pressure. Frontiers in Plant Science, v. 8, p. 1-10, 2017. Disponível: <https://www.frontiersin.org/articles/10.3389/fpls.2017.00054/full>. doi: 10.3389/fpls.2017.00054

VIANA, L. A.; ZAMBOLIM, L.; SOUSA, T. V.; TOMAZ, B. C. Potencial uso de câmera termal acoplada a vant para monitoramento de culturas. Brazilian Journal of Biosystems Engineering, v. 12, n. 3, p. 286-298, 2018. Disponível: <https://seer.tupa.unesp.br/index.php/BIOENG/article/view/692/383>. doi: 10.18011/bioeng2018v12n3p286-298 
VIANA, L. D. A.; TOMAZ, D. C.; MARTINS, R. N.; ROSAS, J. T. F.; DOS SANTOS, F. F. L.; PORTES, M. F. OpticalSensors for PrecisionAgriculture: An Outlook. Journal of Experimental Agriculture International, v. 35, n. 2, p. 1-9, 2019. Disponível: $<$ https://www.journaljeai.com/index.php/JEAl/article/view/30203>. 10.9734/jeai/2019/v35i230203

YANG, S. J.; SUN, M.; YANG, Q. Y.; MA, R. Y.; ZHANG, J. L.; ZHANG, S. B. Two strategies by epiphytic orchids for maintaining water balance: thick cuticles in leaves and water storage in pseudobulbs. AoB Plants, v. 8, plw046, 2016. Disponível: <https://academic.oup.com/aobpla/article/doi/10.1093/aobpla/plw046/2609602>. doi: 10.1093/aobpla/plw046

YAN, W.; ZHONG, Y.; SHANGGUAN, Z. A meta-analysis of leaf gas exchange and water status responses to drought. Sci. Rep., v. 12, n. 6, p. 20917, 2016. Disponível: <https://pubmed.ncbi.nlm.nih.gov/26868055/>. doi: 10.1038/srep20917

ZIA, S.; DU, W.; SPREER, W.; SPOHRER, K.; HE, X.; MÜLLER, J. Assessing crop water stress of winter wheat by thermography under different irrigation regimes in North China Plain. International Journal of Agricultural and Biological Engineering, v. 5, n. 3, p. 24-34, 2012 . Disponível: <https://ijabe.org/index.php/ijabe/article/view/573>. doi: 10.3965/j.ijabe.20120503.00?

ZIA, S.; SOPHRER, K.; DU, W.; SPREER, W.; ROMANO, G.; XIONGKUI, H.; MÜLLER, J. Monitoring physiological responses to water stress in two maize varieties by infrared thermography.International Journal of Agricultural and Biological Engineering, v. 4, n. 3, p. 7-15, 2011. Disponível: <https://ijabe.org/index.php/ijabe/article/view/464/344>. doi: 10.3965/j.issn.19346344.2011.03.007-015

ZOVKO, M.; BORAS, I.; SVAIĆ, Assessing plant water status from infrared thermography for irrigation management. 14th Quantitative Infra Red Thermography Conference, 2529 June 2018, Berlin, Germany. 2018. Disponível: <http://www.qirt.org/archives/qirt2018/papers/050.pdf>. doi: 10.21611/qirt.2018.050 